\title{
Practical Immuno-PET Radiotracer Design Considerations for Human Immune Checkpoint Imaging
}

\author{
Aaron T. Mayer ${ }^{1,2}$, Arutselvan Natarajan ${ }^{2}$, Sydney R. Gordon ${ }^{3-6}$, Roy L. Maute ${ }^{3-6}$, Melissa N. McCracken ${ }^{3-6}$, \\ Aaron M. Ring ${ }^{7}$, Irving L. Weissman ${ }^{3-6}$, and Sanjiv S. Gambhir ${ }^{1,2,8}$ \\ ${ }^{I}$ Department of Bioengineering, Stanford University, Stanford, California; ${ }^{2}$ Department of Radiology, Stanford University, Stanford, \\ California; ${ }^{3}$ Institute for Stem Cell Biology and Regenerative Medicine, Stanford University, Stanford, California; ${ }^{4}$ Ludwig Center for \\ Cancer Stem Cell Research and Medicine, Stanford University, Stanford, California; ${ }^{5}$ Stanford Cancer Institute, Stanford University, \\ Stanford, California; ${ }^{6}$ Department of Pathology, Stanford University, Stanford, California; ${ }^{7}$ Department of Immunobiology, Yale \\ School of Medicine, New Haven, Connecticut; and ${ }^{8}$ Department of Materials Science \& Engineering, Stanford University, \\ Stanford, California
}

\begin{abstract}
Immune checkpoint blockade has emerged as a promising cancer treatment paradigm. Unfortunately, there are still a large number of patients and malignancies that do not respond to therapy. A major barrier to validating biomarkers for the prediction and monitoring of responders to clinical checkpoint blockade has been the lack of imaging tools to accurately assess dynamic immune checkpoint expression. Here, we sought to optimize noninvasive immuno-PET imaging of human programmed death-ligand 1 (PD-L1) expression, in a preclinical model, using a small high-affinity engineered protein scaffold (HAC-PD1). Six HAC-PD1 radiotracer variants were developed and used in preclinical imaging and biodistribution studies to assess their ability to detect human PD-L1 expression in vivo. Radiotracer design modifications included chelate, glycosylation, and radiometal. HACA-PD1 was adopted as the naming convention for aglycosylated tracer variants. NOD scid $\gamma$-(NSG) mice were inoculated with subcutaneous tumors engineered to either be constitutively positive (CT26 hPD-L1) or be negative ( $\triangle \mathrm{mPD}-\mathrm{L} 1 \mathrm{CT} 26)$ for human PD-L1 expression. When the tumors had grown to an average size of $1 \mathrm{~cm}$ in diameter, mice were injected with $0.75-2.25$ $\mathrm{MBq}(\sim 10 \mu \mathrm{g})$ of an engineered radiotracer variant and imaged. At $1 \mathrm{~h}$ after injection, organs were harvested for biodistribution. Of the practical immuno-PET tracer modifications considered, glycosylation was the most prominent design factor affecting tracer uptake, specificity, and clearance. In imaging studies, aglycosylated ${ }^{64} \mathrm{Cu}-\mathrm{NOTA}$-HACA-PD1 most accurately visualized human PD-L1 expression in vivo. We reasoned that because of the scaffold's small size $(14 \mathrm{kDa})$, its pharmacokinetics may be suitable for labeling with the short-lived and widely clinically available radiometal ${ }^{68} \mathrm{Ga}$. At $1 \mathrm{~h}$ after injection, ${ }^{68} \mathrm{Ga}-\mathrm{NOTA}-\mathrm{HACA}-\mathrm{PD} 1$ and ${ }^{68} \mathrm{Ga}-$ DOTA-HACA-PD1 exhibited promising target-to-background ratios in ex vivo biodistribution studies (12.3 and 15.2 tumor-tomuscle ratios, respectively). Notably, all HAC-PD1 radiotracer variants enabled much earlier detection of human PD-L1 expression ( $1 \mathrm{~h}$ after injection) than previously reported radiolabeled antibodies ( $>24 \mathrm{~h}$ after injection). This work provides a template for assessing immuno-PET tracer design parameters and supports the translation of small engineered protein radiotracers for imaging human immune checkpoints.
\end{abstract}

Received May 16, 2016; revision accepted Dec. 2, 2016.

For correspondence or reprints contact: Sanjiv Sam Gambhir, James H. Clark Center, 318 Campus Dr., E153, Stanford, CA 94305.

E-mail: sgambhir@stanford.edu

Published online Dec. 15, 2016.

COPYRIGHT (C 2017 by the Society of Nuclear Medicine and Molecular Imaging.
Key Words: cancer immunotherapy; checkpoint blockade; immunoPET; PD-1; PD-L1

J Nucl Med 2017; 58:538-546

DOI: 10.2967/jnumed.116.177659

I mmune checkpoints that negatively regulate $\mathrm{T}$ cell effector function are now well established to play a role in cancer progression and immune evasion (1-3). Although immune checkpoints typically maintain physiologic self-tolerance, they have been found to be overexpressed in a variety of cancers and to be upregulated by tumors in the presence of infiltrating lymphocytes $(4,5)$. Clinical checkpoint blockade with antibody therapeutics has emerged as a promising treatment paradigm with Ipilimumab (CTLA-4; BristolMyers Squibb), Pembrolizumab (PD-1 [programmed cell death-1]; Merck), and Nivolumab (PD-1; Bristol-Myers Squibb) all having been recently approved by the Food and Drug Administration for the treatment of advanced melanoma (6-10). Success has spurred further clinical trials for several malignancies.

Unfortunately, not all patients respond to immune checkpoint blockade. Challenges exist including appropriate trial design, patient selection, dose determination, and complex pharmacokinetics (11). Several potential biomarkers have emerged including programmed death-ligand 1 (PD-L1) or PD-1 expression (12-21). Although PD-L1 expression has been linked to poor prognoses and better therapeutic responses, its true predictive value is still unknown. Biomarker validation has been confounded by ex vivo results from histologic studies using different antibodies and positive or negative staining thresholds (15). There is an explicit need for molecular imaging tools that can noninvasively capture and quantify the spatiotemporal expression profiles of cancer-associated immune checkpoints.

Immuno-PET imaging can provide a means to noninvasively assess antibody drug pharmacokinetics and the expression of cell surface markers of disease (22). Here we would like to broaden the conventional definition of immuno-PET as imaging with radiolabeled antibodies to include imaging with engineered antibody fragments and protein binders that target specific immune clusters of differentiation markers. Antibody-based imaging agents have several advantages including their naturally high avidity, antigen specificity, and ease of production. For these reasons, early attempts at 
immune checkpoint imaging have used antibody scaffolds including $3{ }^{111}$ In radiotracers directed against murine and human PD-L1 for SPECT imaging of breast and non-small cell lung cancer (23-25). Our laboratory has also developed a murine anti-PD1 monoclonal antibody radiolabeled with ${ }^{64} \mathrm{Cu}$ for imaging PD-1 expression on tumor-infiltrating lymphocytes (26). Although it has been shown that antibodies' large size and Fc region contribute to enhanced blood half-lives and high tumor uptake, this poses a unique challenge for imaging because long circulation times contribute to high background signal and nonspecific uptake. In all of these studies, immune checkpoint expression in tumors was not clearly visualized until longer than $24 \mathrm{~h}$ after injection.

The engineering of small, high-affinity protein binders can potentially overcome the limitations of immuno-PET imaging with antibodies and provide an accurate means to assess biomarkers for clinical checkpoint blockade. Using the ectodomain of wild-type PD-1 protein, we previously affinity-matured a 14-kDa PD-L1 binder with a $100 \mathrm{pM}$ dissociation constant, which exhibited high immunoreactivity in vitro and in pilot in vivo PET imaging studies. This protein, termed high-affinity consensus (HAC) PD1, is to our knowledge the first engineered binder to be used for human PD-L1 immune checkpoint imaging (27). As a therapeutic, HAC-PD1 significantly enhanced survival in mice bearing large PD-L1-positive tumors compared with treatment with anti-PD-L1 monoclonal antibodies. Here, we optimize HAC-PD1 for imaging of in vivo human PD-L1 expression at early time points, by modifying common immuno-PET design parameters including chelate, glycosylation, and radiometal.

\section{MATERIALS AND METHODS}

\section{General Procedures}

Animal studies were performed in compliance with approval from the Administrative Panel on Laboratory Animal Care at Stanford University.

\section{Cell Lines and Animal Models}

The murine colon carcinoma cell line CT26 (CRL. 2639) was obtained from the American Type Culture Collection. CT26 genetic variants expressing human PD-L1 were generated using the methods previously reported (27). All cells were grown in RPMI supplemented with $10 \%$ fetal bovine serum, glutamine $(2 \mathrm{mmol} / \mathrm{L})$, penicillin $(100$ units $/ \mathrm{mL})$, streptomycin $(100 \mu \mathrm{g})$, and fungizone $(0.25 \mu \mathrm{g} / \mathrm{mL})$ and maintained in a humidified, $5 \% \mathrm{CO}_{2}$ incubator at $37^{\circ} \mathrm{C}$. All medium and additives were obtained from Invitrogen Corporation. In vivo PET studies were performed using Nod.Cg-Prkdc.scid.IL2rg.tm1Wj1/SzJ (NSG) mice (age, 6-8 wk) obtained from in-house breeding and implanted with CT26 cell line variants.

\section{Reagents and Instruments}

1,4,7,10-tetraazacyclododecane-1,4,7-tris-acetic acid-10-maleimidoethylacetamide (DOTA-maleimide) and 1,4,7-triazacyclononane1,4-bis-acetic acid-7-maleimidoethylacetamide (NOTA-maleimide) were purchased from Macrocyclics. ${ }^{64} \mathrm{CuCl} 2$ (half-life, $12.7 \mathrm{~h}$; radionuclide purity $>99 \% ; 2-3 \mathrm{GBq} / \mathrm{ml}$ in $0.1 \mathrm{M}$ hydrochloric acid) was obtained from the University of Wisconsin. The ${ }^{68} \mathrm{GaCl} 3$ (half-life, $67.6 \mathrm{~min}$; radionuclide purity $>95 \%$ ) was eluted from a ${ }^{68} \mathrm{Ge} /{ }^{68} \mathrm{Ga}$ generator (IGG-100; Eckert \& Ziegler Isotope Products) using 3-5 mL of $0.1 \mathrm{M} \mathrm{HCl}$. To obtain pure ${ }^{68} \mathrm{Ga}$, the elution was passed through a cation exchange cartridge to trap the ${ }^{68} \mathrm{Ga}$ ions alone. The trapped pure ${ }^{68} \mathrm{GaCl} 3$ was eluted from the cartridge using a concentrated $\mathrm{NaCl} / \mathrm{HCl}$ solution and yielded $444-555 \mathrm{MBq}(12-15 \mathrm{mCi})$ of ${ }^{68} \mathrm{GaCl} 3$. All other reagents were obtained from Sigma-Aldrich unless otherwise stated. High-performance liquid chromatography (HPLC) was performed on a HPLC-Ultimate 2000 with a Phenomenex SEC2000 LC column $(300 \times 7.8 \mathrm{~mm})$ with $5 \mu \mathrm{m}$, hydrophilic bonded silica support of 400 - $\AA$ pore size with an ultraviolet detector and a radioactivity detector. Mass spectrometry (AB SCIEX TOF/TOF 5800) was performed at Stanford University and operated in linear mode with sinapinic acid as the matrix.

\section{Synthesis of Immuno-PET Binders}

HAC_N91C expression and purification have previously been described (27). The putative glycosylation sites N49D and N58D were mutated to aspartic acid residues to form the aglycosylated HACA binder. The PET precursors were prepared by conjugating HAC or aglycosylated HACA binders with DOTA-maleimide or NOTA-maleimide to generate 4 precursors of HAC-DOTA, HAC-NOTA, HACA-DOTA, and HACA-NOTA. Briefly, HAC or HACA binders were purified by size-exclusion chromatography with $0.1 \mathrm{M}$ sodium phosphate buffer $(\mathrm{pH} 7)$ at $1 \mathrm{~mL} / \mathrm{min}$ and concentrated to approximately $1-2 \mathrm{mg} \mathrm{mL}^{-1}$ using an Amicon $7 \mathrm{kDa}$ centrifugal Ultra-2 filter. Concentrated HAC or HACA $(0.01 \mathrm{mM})$ was then mixed with a solution of DOTA-maleimide or NOTA-maleimide in $80 \%$ dimethyl sulfoxide in water at a 5-fold molar excess. The $\mathrm{pH}$ of the reaction mixture was adjusted to 6.5-7.0, and then the mixture was incubated in a dark room for $60 \mathrm{~min}$ at $37^{\circ} \mathrm{C}$. The precursor was purified from the excess DOTA or NOTA chelates by SEC2000 HPLC using $0.1 \mathrm{M}$ ammonium acetate buffer $(\mathrm{pH} \mathrm{5.5)}$ as the mobile phase eluted at $1 \mathrm{~mL} / \mathrm{min}$. The precursors were concentrated to approximately $1 \mathrm{mg} / \mathrm{mL}$. The number of chelates coupled per antibody was estimated with matrix-assisted laser desorption/ionization time-of-flight mass spectrometry by comparison with unmodified HAC and DOTA- or NOTA-modified HAC or HACA.

\section{Synthesis and Purification of PET Tracers}

Ammonium acetate buffer (0.1 M, pH 5.5) was added to 1.5 -mL vials containing $0.2 \mathrm{mg}$ of either DOTA-HAC, NOTA-HAC, DOTA-HACA, or NOTA-HACA. To each of these vials, $0.5-1 \mathrm{~mL}$ of ${ }^{68} \mathrm{Ga}$ isotope $(370-444 \mathrm{MBq})$ or ${ }^{64} \mathrm{Cu}(60-300 \mathrm{MBq})$ were added and kept at $37^{\circ} \mathrm{C}$. After $45 \mathrm{~min}, 0.1 \mathrm{~mL}$ of aqueous ethylenediaminetetraacetic acid solution $(0.1 \mathrm{M}, \mathrm{pH} 7.0)$ was added to the reaction mixture for $15 \mathrm{~min}$ to scavenge unchelated ${ }^{64} \mathrm{Cu}$ or ${ }^{68} \mathrm{Ga}$ in the reaction mixture. The radiotracers were purified by a SEC2000 column in phosphate-buffered saline (PBS) (pH 7.4) at a flow rate of $1.0 \mathrm{~mL} / \mathrm{min}$. The radiotracer peak (retention time, $9.5 \mathrm{~min}$ ) was collected and concentrated using an Amicon Ultra-15 7kDa cutoff device and centrifuged at 3,000 g for $15 \mathrm{~min}$. The final product was filtered through a $0.2-\mu \mathrm{m}$ filter into a sterile vial. The ${ }^{64} \mathrm{Cu}-\mathrm{HAC} /{ }^{68} \mathrm{Ga}-\mathrm{HAC}$ radiochemical purity was analyzed by thinlayer chromatography as well as SEC2000 radio-HPLC.

\section{Immunoreactivity}

To assess the immunoreactive fraction of the HAC/HACA-PD1 variants, the constructs were tested using hPDL1 $(+)$ cells, hPDL1 $(+)$ cells preblocked with HAC-PD1, and control hPDL1(-) cells. Each of the tracers $(5 \mathrm{nmol} / \mathrm{L} ; 4-6 \mathrm{MBq} / \mathrm{nmol})$ was mixed separately with $2.5 \times 105$ cells in $0.1 \mathrm{~mL}$ of PBSA (PBS supplemented with $1 \%$ bovine serum albumin) in triplicate and incubated for $60 \mathrm{~min}$. One hour later, the solutions were centrifuged ( $300 \mathrm{~g}$ for $5 \mathrm{~min}$ ), resuspended, and washed twice with ice-cold PBSA before the supernatant was removed. Cells were then pelleted by centrifugation, and counting was performed for the ${ }^{64} \mathrm{Cu}$ or ${ }^{68} \mathrm{Ga}$ activity associated with the cell pellet. The activity was counted in a $\gamma$-counter (1470 WIZARD Automatic Gamma Counter; Perkin Elmer). The count data were background corrected and compared with the total number of counts in control samples.

\section{Animal Studies}

The average weight of the mice was $20.0 \pm 2.0 \mathrm{~g}$. Groups of NSG mice were inoculated with subcutaneous hPD-L1-positive tumors in 


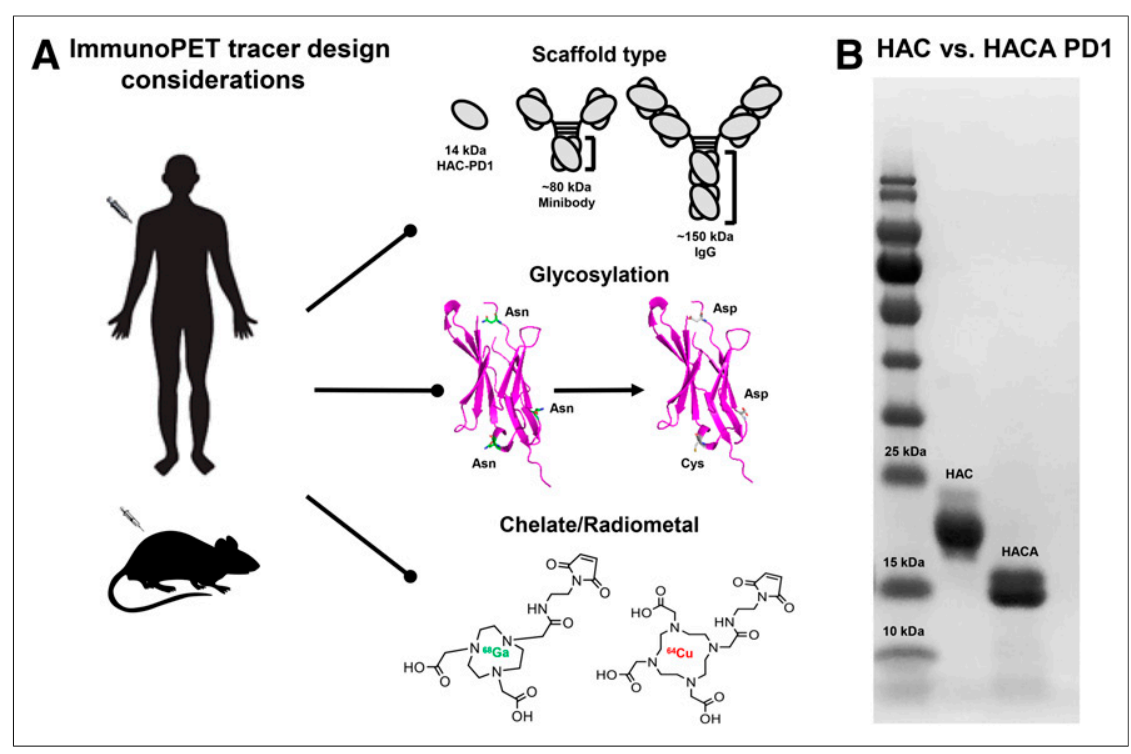

FIGURE 1. Immuno-PET tracer design considerations. (A) Common engineering parameters include scaffold, glycosylation, chelate, and radiometal. Scaffold type: size comparison of 14-kD HAC-PD1 to other radiotracer scaffolds. Glycosylation: the HAC-PD1 glycosylated form of radiotracer has 3 asparagine residues in its backbone. These sites were mutated to aspartic acid residues to develop aglycosylated HACA-PD1 variant. Chelate: either DOTA- or NOTA-maleimide chelates were conjugated to HAC or HACA-PD1 scaffold via cysteine residue in its backbone. Radiometal: precursors were radiolabeled with ${ }^{64} \mathrm{Cu}$ or ${ }^{68} \mathrm{Ga}$ to formulate final radiotracers. (B) Sodium dodecyl sulfate polyacrylamide gel electrophoresis gel showing size differences between HAC-PD1 glycosylated protein and aglycosylated HACA-PD1 variant.

their right shoulder and hPD-L1-negative tumors in their left shoulder (Supplemental Table 1; supplemental materials are available at http:// jnm.snmjournals.org). Single- and dual-tumor-bearing mice were imaged at $1 \mathrm{~h}$ using small-animal PET/CT at the Stanford small-animal imaging center. Mice received ${ }^{64} \mathrm{Cu}$ or ${ }^{68} \mathrm{Ga}$ radiotracers $(200 \mu \mathrm{L}$, corresponding to $0.7-3.7 \mathrm{MBq}, 10-15 \mu \mathrm{g}$ of DOTA-/NOTA-HAC) via lateral tail vein injection.

\section{Small Animal Immuno-PET/CT Imaging}

PET/CT imaging was performed on the Inveon Small-Animal Multimodality PET/CT System (Preclinical Solutions; Siemens Healthcare Molecular Imaging). Instrument specifications and imaging methods have previously been described (26). PET images were reconstructed with the 2-dimensional ordered-subset expectation maximization algorithm. PET/CT images and 3-dimensional regions of interest (ROIs) were computed using the Inveon Research Workplace software (Preclinical Solutions). The mean pixel value within the ROI volume was converted to radioactivity concentration in counts per milliliter per minute (cpm) using a predetermined conversion factor. The percentage injected dose per gram of tissue (\% ID/g) was estimated by dividing each tissue's cpm obtained from the ROI by the injected dose.

\section{Biodistribution}

Tracer uptake in each organ was evaluated by ex vivo biodistribution of dual-tumor-bearing NSG mice. Biodistribution studies were performed $1 \mathrm{~h}$ after injection of radiotracer, and organs were isolated, rinsed in PBS, and dried in air before finally being weighed and counted with a gamma-counter (Cobra II Auto-Gamma counter; Packard Biosciences, Co.). All data were decay corrected. The $\% \mathrm{ID} / \mathrm{g}$ for each tissue sample was calculated by normalizing to the total activity injected.

\section{Statistical Analysis}

The unpaired Student $t$ test was used for data comparisons. $P$ values of less than 0.05 were considered statistically significant.

\section{RESULTS}

\section{Preparation of Antihuman \\ PD-L1 Radiotracers}

Six novel immuno-PET radiotracers were engineered on the HAC-PD1 backbone: ${ }^{64} \mathrm{Cu}-\mathrm{DOTA}-\mathrm{HAC}-\mathrm{PD} 1,{ }^{64} \mathrm{Cu}-\mathrm{NOTA}-\mathrm{HAC}-$ PD1, ${ }^{64} \mathrm{Cu}-\mathrm{NOTA}-\mathrm{HACA}-\mathrm{PD} 1,{ }^{68} \mathrm{Ga}-\mathrm{NOTA}-$ HAC-PD1, ${ }^{68}$ Ga-NOTA-HACA-PD1, and ${ }^{68}$ Ga-DOTA-HACA-PD1. We chose not to pursue the remaining 2 permutations, ${ }^{64} \mathrm{Cu}$ DOTA-HACA-PD1 and ${ }^{68} \mathrm{Ga}-D O T A-H A C-$ PD1, based on data from literature suggesting NOTA is the favored chelate for use with ${ }^{64} \mathrm{Cu}(28)$ and pilot data suggesting HACA is the preferred binder, respectively. Engineering design considerations included chelate, glycosylation, and radiometal (Fig. 1A). A major advancement in this work was the engineering of HACA-PD1, the aglycosylated tracer variant. Gel electrophoresis confirmed the successful elimination of glycans in the protein scaffold (Fig. 1B). The engineered free cysteine residue (N91C) enabled site-specific conjugation of, at maximum, 1 chelate per protein, and this was confirmed via mass spectrometry. The mean specific activities of ${ }^{64} \mathrm{Cu}$-DOTA and ${ }^{64} \mathrm{Cu}$ NOTA variants ranged from $4,843.8$ to $6,199.5 \mathrm{MBq} / \mu \mathrm{mol}$, whereas ${ }^{68} \mathrm{Ga}$-DOTA and ${ }^{68} \mathrm{Ga}$-NOTA variants provided 1,455.6-2,162.9 MBq/ $\mu$ mol. Chemical purity was measured by SEC-HPLC to be greater than $85 \%$, whereas radiochemical purity was on average $87 \%$ for all variants (Supplemental Fig. 1; Supplemental Table 1).

\section{Optimized Immune Checkpoint Imaging with Engineered ${ }^{64} \mathrm{Cu}$-Radiolabeled HAC-PD1 Variants}

Human PD-L1 expression in positive tumors was clearly visualized as early as $1 \mathrm{~h}$ after injection of the ${ }^{64} \mathrm{Cu}$-DOTAHAC-PD1 tracer in both dual- and single-tumor models (Fig. 2A; Supplemental Fig. 2; Supplemental Video 1). ROI analysis of the dual-tumor model revealed that tumors positive for human PDL1 exhibited significantly higher tracer uptake $(1.6 \pm 0.3 \% \mathrm{ID} / \mathrm{g})$ than PD-L1-negative tumors $(0.9 \pm 0.1 \% \mathrm{ID} / \mathrm{g})$, whereas in the single-tumor model, human PD-L1-positive tumors had a greater than 2-fold-higher tracer uptake than mice bearing positive tumors preblocked with $500 \mu \mathrm{g}$ of cold HAC-PD1 (Supplemental Fig. 2). Static scans at 1, 2, 4, 18, and $24 \mathrm{~h}$ after injection revealed that uptake in the tumor slightly increased over time whereas the tracer cleared slowly through the kidneys and bladder (Supplemental Fig. 3). The tracer also exhibited a high nonspecific uptake in the salivary glands and lymph nodes of the head and neck as well as the spleen. The ${ }^{64} \mathrm{Cu}-\mathrm{NOTA}-\mathrm{HAC}-\mathrm{PD} 1$ radiotracer exhibited a significantly higher uptake in human PD-L1-positive tumors (ROI $3.3 \pm 0.85 \% \mathrm{ID} / \mathrm{g}$ ) than ${ }^{64} \mathrm{Cu}-\mathrm{DOTA}-\mathrm{HAC}-\mathrm{PD} 1$ (ROI $1.6 \pm 0.3 \% \mathrm{ID} / \mathrm{g}$ ), but its ability to distinguish PD-L1-positive tumors from -negative tumors was slightly diminished (Fig. 2B; Supplemental Fig. 2; Supplemental Video 2). ${ }^{64} \mathrm{Cu}-\mathrm{NOTA}-\mathrm{HAC}-\mathrm{PD} 1$ also exhibited a significantly higher background signal, especially in the heart and salivary glands whereas a slightly lower signal was seen in the liver. Imaging with aglycosylated ${ }^{64} \mathrm{Cu}$-NOTA-HACA-PD1 provided the best visualization of human PD-L1-positive tumors (Fig. 2C; Supplemental 


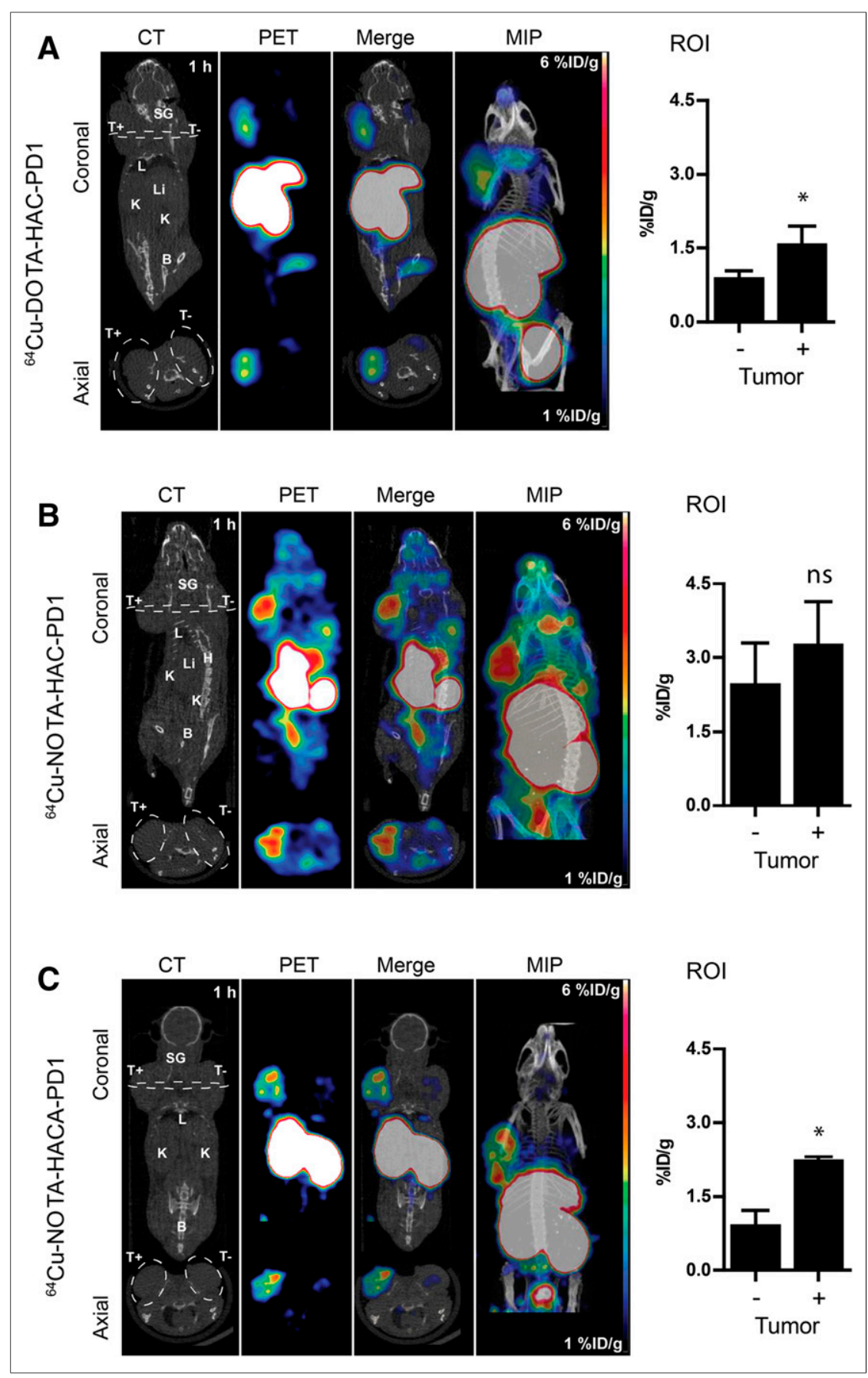

FIGURE 2. Human PD-L1 immune checkpoint imaging using ${ }^{64} \mathrm{Cu}$. Comparison of ${ }^{64} \mathrm{Cu}$-DOTAHAC-PD1 (A), ${ }^{64} \mathrm{Cu}-\mathrm{NOTA}-\mathrm{HAC}-\mathrm{PD} 1$ (B), and ${ }^{64} \mathrm{Cu}-\mathrm{NOTA}-\mathrm{HACA}-\mathrm{PD} 1$ (C) immuno-PET images acquired at $1 \mathrm{~h}$ after injection $(\sim 1.85 \mathrm{MBq} / 10 \mu \mathrm{g} / 200 \mu \mathrm{L})$ of NSG mice bearing dual subcutaneous tumors in shoulders (right $=\mathrm{hPD}-\mathrm{L} 1+$; left $=\mathrm{hPD}-\mathrm{L} 1-$ ). Panels left to right show representative coronal and axial cross sections of CT, PET, and merged PET/CT images with maximum-intensity projection (MIP). White dashed line on coronal image shows demarcation of axial cross-section; white dashed line on axial image shows tumor boundary. Scale bars represent 1 (blue)-6 (red) \%ID/g. Tumor uptake was quantified using ROI analysis (right panel) without PVC. Error bars represent $\mathrm{SD}$. ${ }^{*} \mathrm{P}<0.05$, Student $t$ test. $\mathrm{B}=$ bladder; $\mathrm{H}=$ heart; $\mathrm{K}=$ kidney; $\mathrm{L}=$ lung; $\mathrm{Li}=$ liver; $\mathrm{ns}=$ not significant; SG = salivary gland; T+ = hPD-L1-positive tumor; T- = hPD-L1-negative tumor.

Video 3). ${ }^{64} \mathrm{Cu}-\mathrm{NOTA}-\mathrm{HACA}-\mathrm{PD} 1$ maintained a high PD-L1positive tumor uptake while exhibiting a noticeably decreasing background signal. In particular, ${ }^{64} \mathrm{Cu}-\mathrm{NOTA}-\mathrm{HACA}-\mathrm{PD} 1 \mathrm{had} \mathrm{a}$ dramatically reduced nonspecific uptake in the glands of the head and neck and showed a significantly reduced spleen and liver signal. PD-L1-positive tumor uptake was $2.3 \pm$ $0.1 \% \mathrm{ID} / \mathrm{g}$ compared with a $0.9 \pm 0.3 \% \mathrm{ID} / \mathrm{g}$ uptake in PD-L1-negative tumors as measured by ROI in the dual-tumor model.

\section{Quantitative ${ }^{64} \mathrm{Cu}$ \\ Immuno-PET Imaging}

To assess the accuracy of ${ }^{64} \mathrm{Cu}$ immunoPET imaging, ROI values were quantitated and compared with ex vivo $\gamma$-counter measures of tumor uptake. \%ID/g values calculated via ROI analysis corresponded closely with those obtained via ex vivo $\gamma$-counting (without partial-volume correction (PVC), $\mathrm{y}=0.68 \mathrm{x} \pm 0.19 ; r^{2}=0.87$; with PVC, $\mathrm{y}=0.744 \mathrm{x} \pm 0.25 ; r^{2}=0.86$ ). $\mathrm{PVC}$ reduced the percentage error in these measurements (ROI mean \%error $=-20.95 \% \pm 4.72 \%$; PVC-corrected mean \%error $=-11.49 \% \pm 5.52 \%)$ and revealed that the ROI values slightly underestimate the true uptake, especially at smaller tumor sizes (Fig. 3).

\section{Biodistribution of ${ }^{64} \mathrm{Cu}$-Radiolabeled HAC-PD1 Variants}

${ }^{64} \mathrm{Cu}$-DOTA-HAC-PD1 biodistribution results corroborated with the images and ROI analysis in both the single- and the dual-tumor models (Fig. 4A; Supplemental Tables 2-4). PD-L1-positive tumors exhibited a 6.4 times and 6.8 times higher tracer uptake than blood and muscle, respectively, but some organs such as the spleen $(4.0 \pm 3.1 \% \mathrm{ID} / \mathrm{g})$ exhibited a high nonspecific uptake. Because of its $14-\mathrm{kDa}$ size, the ${ }^{64} \mathrm{Cu}$-DOTA-HAC-PD1 tracer primarily underwent renal clearance, evidenced by a high kidney $(113 \pm 15.7$ $\% \mathrm{ID} / \mathrm{g}$ ) signal at $1 \mathrm{~h}$ after injection, although, some hepatobiliary clearance or transchelation via proteins in the liver $(19.8 \pm 2.5 \% \mathrm{ID} / \mathrm{g})$ was also observed. ${ }^{64} \mathrm{Cu}-\mathrm{NOTA}-\mathrm{HAC}-\mathrm{PD} 1$ ex vivo biodistribution supports the high tumor uptake and background signal observed during the imaging (Fig. 4B; Supplemental Tables 2-4). Human PD-L1-positive tumorto-background ratios were subsequently reduced compared with ${ }^{64} \mathrm{Cu}$-DOTA-HACPD1, exhibiting only a 3.7 times and 3.4 times higher tumor uptake than blood and muscle, respectively. Overall, the biodistribution profile was the most favorable for ${ }^{64} \mathrm{Cu}$-NOTA-HACA-PD1 of the ${ }^{64} \mathrm{Cu}$ immuno-PET radiotracer variants, with a higher signal being observed in PD-L1-positive tumors than all other organs in the uptake panel. This increase in specific uptake was reflected in higher tumor-to-background ratios for most organs, 


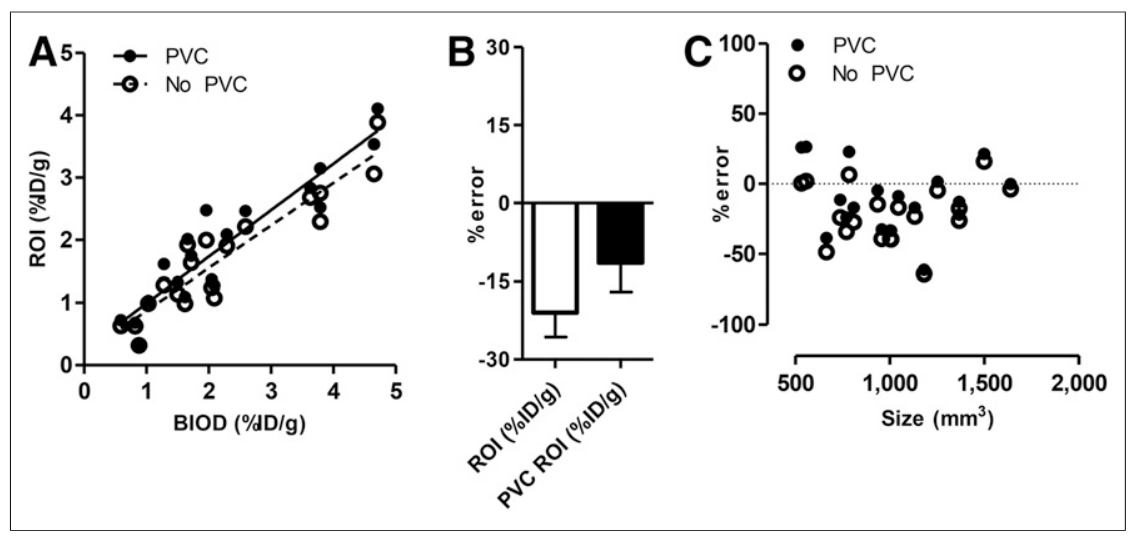

FIGURE 3. Quantitative immuno-PET image analysis. (A) Correlation was assessed between tumor radiotracer uptake (\%ID/g) calculated via $\mathrm{ROI}$ analysis vs. BIOD with $\mathrm{PVC}$ (solid line; $\mathrm{y}=$ $0.744 \mathrm{x} \pm 0.25 ; r^{2}=0.86$ ) and without PVC (dashed line; $\mathrm{y}=0.68 \mathrm{x} \pm 0.19 ; r^{2}=0.87$ ). (B) Mean percentage error for ROI vs. PVC ROI. (C) Percentage error was measured as function of tumor size $\left(\mathrm{mm}^{3}\right)$.

notably a 10.3 times ratio for positive tumor to muscle (Fig. 4C; Supplemental Tables 3 and 4).

\section{Biodistribution of ${ }^{68} \mathrm{Ga}-$ Radiolabeled HAC-PD1 Variants}

${ }^{68} \mathrm{Ga}-\mathrm{NOTA}-\mathrm{HAC}-\mathrm{PD} 1 \mathrm{exhibited} \mathrm{favorable} \mathrm{imaging} \mathrm{and} \mathrm{biodis-}$ tribution properties in pilot studies with the single-tumor model (Supplemental Fig. 4A; Supplemental Table 2). Radiolabeling the aglycosylated variant with ${ }^{68} \mathrm{Ga}$ to formulate ${ }^{68} \mathrm{Ga}$-NOTA-HACA-PD1 resulted in a further enhanced tracer uptake in human PD-L1positive tumors $(3.8 \pm 1.6 \% \mathrm{ID} / \mathrm{g}$ ) (Fig. 5A; Supplemental Tables 3 and 4). This absolute increase in target tumor uptake was accompanied by low nonspecific uptake in other organs, resulting in favorable tumor-to-background ratios (12.3×, muscle). At this time point, a substantial quantity of tracer still remained in circulation, as evidenced by high signal in the blood (8.7 \pm $4.7 \% \mathrm{ID} / \mathrm{g})$ and low renal clearance $(20.9 \pm 15.4 \% \mathrm{ID} / \mathrm{g})$, suggesting this variant may benefit from imaging at slightly later time points and further studies testing its serum stability. ${ }^{68} \mathrm{Ga}-$ DOTA-HACA-PD1 exhibited the highest observed target tumorto-background ratios for the largest number of organs (Fig. 5B; Supplemental Tables 3 and 4) including muscle $(15.2 \times)$, lungs $(6.3 \times)$, spleen $(52 \times)$, pancreas $(7.8 \times)$, small intestine $(9.2 \times)$, large intestine $(5.2 \times)$, bone $(6.8 \times)$, and brain $(68.2 \times)$. Preliminary imaging results with ${ }^{68} \mathrm{Ga}-\mathrm{DOTA}-\mathrm{HACA}-\mathrm{PD} 1$ revealed a high tumor signal and low off-target accumulation in the liver, spleen, and salivary glands (Supplemental Fig. 5B), correlating well with the findings from the biodistribution study.

\section{Effects of Chelate, Glycosylation, and Radiometal}

Heat maps were generated to compare the biodistribution effects of new radiotracer variants compared with ${ }^{64} \mathrm{Cu}$-DOTAHAC-PD1 or the effects of engineering design parameters such as chelate, glycosylation, and radiometal in the dual-tumor model (Fig. 6). Switching the chelate from DOTA to NOTA for ${ }^{64} \mathrm{Cu}$ variants generally resulted in increases not only in tumor uptake but also in all other organs as well. This was due in part to longer circulation time, marked by higher signal in the blood at $1 \mathrm{~h}$. Interestingly, performing the switch from DOTA to NOTA with the ${ }^{68} \mathrm{Ga}$ variants had a nearly identical effect. Despite these differences observed with chelate and radiometal swaps, all glyco- sylated tracers exhibited similar heat map profiles when compared with the original variant. The heat maps reveal that aglycosylation had the largest impact on tracer biodistribution. Aglycosylated variants had a high frequency of hot spots in the muscle, blood, heart, spleen, and liver, indicating that changes in this design parameter substantially affected uptake in these organs. Although using an aglycosylated radiotracer led to slightly longer blood circulation, it resulted in highly favorable decreases in off-target tissue accumulation while increasing target tumor uptake.

\section{DISCUSSION}

Here we assessed practical immunoPET radiotracer design modifications and their effects on human PD-L1 immune checkpoint imaging. We sought to optimize engineering design parameters including chelate, glycosylation, and radiometal to develop a noninvasive molecular imaging tool for eventual monitoring of clinical checkpoint blockade.

Common immuno-PET radiotracer scaffolds range from fulllength antibodies to small engineered proteins. Although radiolabeled antibodies are a powerful tool in the drug development process, antibody-based immuno-PET radiotracers have several clinical drawbacks including long clearance times and high background signal, which require patients to return to the hospital several days after radiotracer injection for imaging $(29,30)$. Furthermore, we have recently shown that anti-PD-L1 antibodies have limited tumor penetration (31) and that the Fc region of anti-PDL1 monoclonal antibodies can mediate unintended $\mathrm{T}$ cell depletion (27). Meanwhile, advances in protein engineering have enabled the development of binders with pharmacokinetic properties more favorable for PET imaging at early time points. Small, high-affinity protein binders exhibiting rapid clearance and high specific uptake are compatible with short-lived isotopes and therefore may impart less radiation dose. For these reasons, we chose HAC-PD1, a 14-kD protein with high affinity to human PD-L1, as our immuno-PET scaffold.

Matching chelates to radiometals is an important consideration because the structure and physical properties of the complex have been shown to have a large impact on the overall immuno-PET tracer biodistribution (32). In this work, we conjugated a free cysteine residue in the HAC-PD-1 backbone to either DOTA or NOTA chelators using site-specific thiol-maleimide chemistry. When the same HAC-PD1 vector radiolabeled with ${ }^{64} \mathrm{Cu}$ was used, NOTA conjugation led to a slightly different uptake and clearance profile than DOTA. ${ }^{64} \mathrm{Cu}-\mathrm{NOTA}-\mathrm{HAC}-\mathrm{PD} 1$ exhibited longer blood circulation and higher human PD-L1-positive tumor signal at the 1 -h time point. Both the ${ }^{64} \mathrm{Cu}$-DOTA-HAC-PD1 and the ${ }^{64} \mathrm{Cu}-\mathrm{NOTA}-\mathrm{HAC}-\mathrm{PD} 1$ variants exhibited high splenic and glandular uptake in the head and neck region as well as nonspecific signal in the liver. This was likely due to uptake and clearance via the reticular endothelial system and nonspecific binding by proteins in the liver and intestinal tract that can transchelate or bind free copper. 


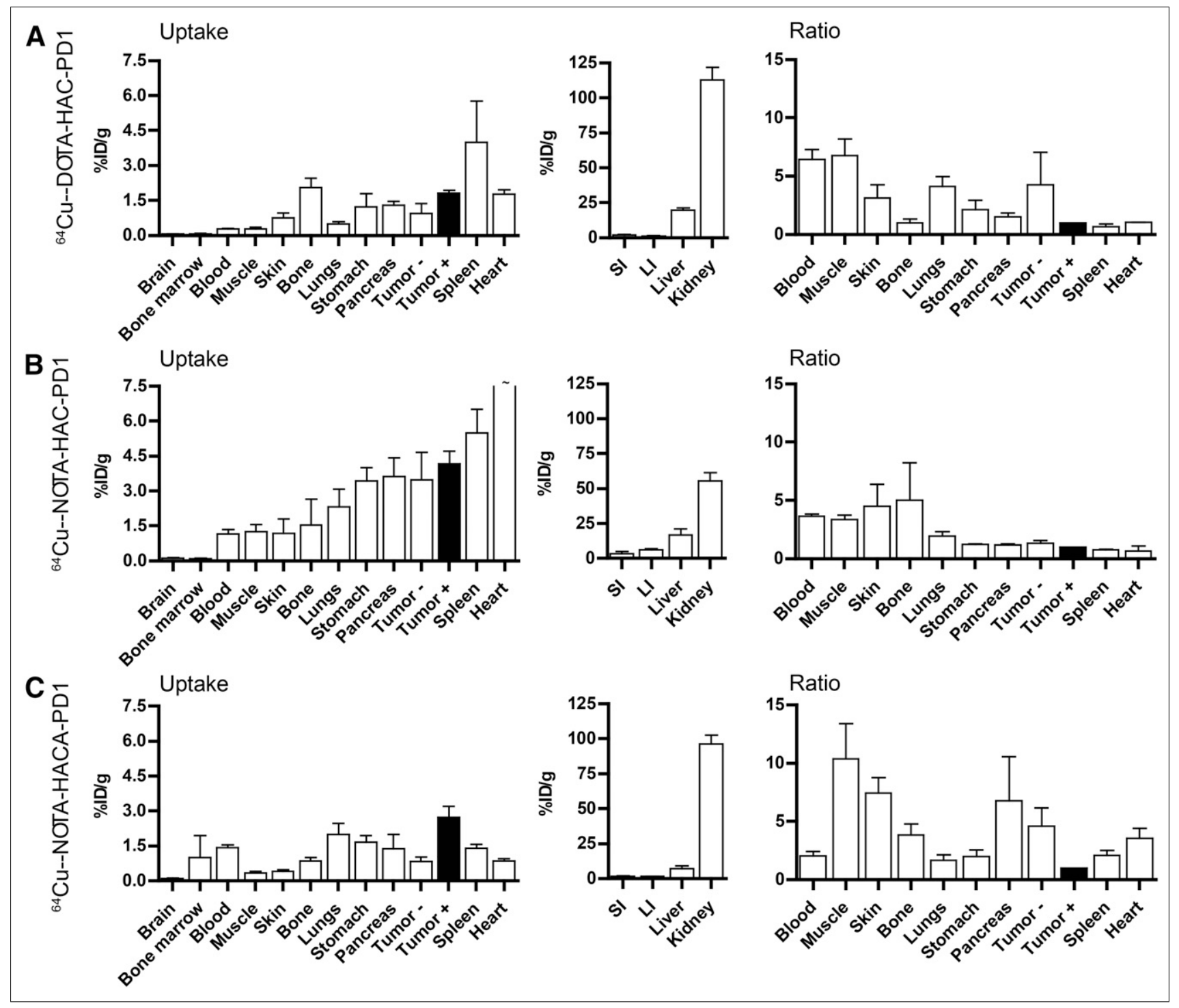

FIGURE 4. ${ }^{64} \mathrm{Cu}$ immuno-PET radiotracer biodistribution. After $1 \mathrm{~h}$ after injection $(\sim 1.85 \mathrm{MBq} / 10 \mu \mathrm{g} / 200 \mu \mathrm{L})$ of ${ }^{64} \mathrm{Cu}-\mathrm{DOTA}-\mathrm{HAC}-\mathrm{PD} 1$ (A), ${ }^{64} \mathrm{Cu}-$ NOTA-HAC-PD1 (B), or ${ }^{64} \mathrm{Cu}-\mathrm{NOTA}-H A C A-P D 1$ (C), organs were harvested and radiotracer uptake was measured with a $\mathrm{y}$-counter. Biodistribution profiles represent mean \pm SEM decay-corrected \%ID/g immuno-PET tracer uptake in tissues and clearance organs. Target-to-background ratios were calculated by dividing hPD-L1-positive tumor radiotracer uptake (\%ID/g) by nonspecific tracer uptake (\%ID/g) in each organ. Data are reported as mean \pm SEM target-to-background ratio for each cohort ( data off-scale, refer to Supplemental Tables 2 and 3 ). $\mathrm{LI}=\mathrm{large}$ intestine; $\mathrm{SI}=\mathrm{small}$ intestine; $\mathrm{T}+$ = tumor positive; $\mathrm{T}-\mathrm{=}$ tumor negative.

We hypothesized that our $N$-glycosylated HAC-PD1 radiotracer may undergo pattern recognition and clearance by mannose-binding proteins, which can be found as cell surface receptors on macrophages and dendritic cells in the spleen as well as hepatic endothelial cells in the liver (33). To prevent glycosylation of HAC, we mutated the asparagine residues in the NxS/T motifs found in the HAC-PD1 scaffold to aspartic acid. Glycosylation is a complex process and certain carbohydrate motifs can result in drastically different biologic outcomes. Imaging revealed highly specific uptake of the aglycosylated ${ }^{64} \mathrm{Cu}-\mathrm{NOTA}-\mathrm{HACA}-$ PD1 radiotracer in tumors expressing human PD-L1 and elimination of nonspecific glandular signal in the head and neck. As expected, ${ }^{64} \mathrm{Cu}$-NOTA-HACA-PD1 exhibited less splenic and hepatobiliary uptake. Here our findings were in line with the general notions that NOTA is the preferred chelate for use with ${ }^{64} \mathrm{Cu}$ and that glycosylation can lead to nonspecific uptake. ${ }^{64} \mathrm{Cu}-\mathrm{NOTA}-$ HACA-PD1 was the best performing ${ }^{64} \mathrm{Cu}$ tracer variant. Although aglycosylation decreased nonspecific tissue uptake and improved image quality, it increased blood circulation times. Further studies should seek to optimize blood clearance time and seek to understand how much of the blood signal may be from free radiometal, to take full advantage of the improved pharmacologic profiles of the HACA variants.

Because radiometal selection is also known to affect tracer biodistribution (28), we tested DOTA and NOTA variants radiolabeled with ${ }^{68} \mathrm{Ga}$. For example, ${ }^{64} \mathrm{Cu}$ is known to accumulate in the liver whereas ${ }^{68} \mathrm{Ga}$ is known to accumulate in the bone (28). In those lines, ${ }^{68} \mathrm{Ga}$ variants tested in this study significantly reduced liver signal when compared with ${ }^{64} \mathrm{Cu}$ variants. In pilot data using the single-tumor model, ${ }^{68} \mathrm{Ga}$-NOTA-HAC-PD1 showed favorable 


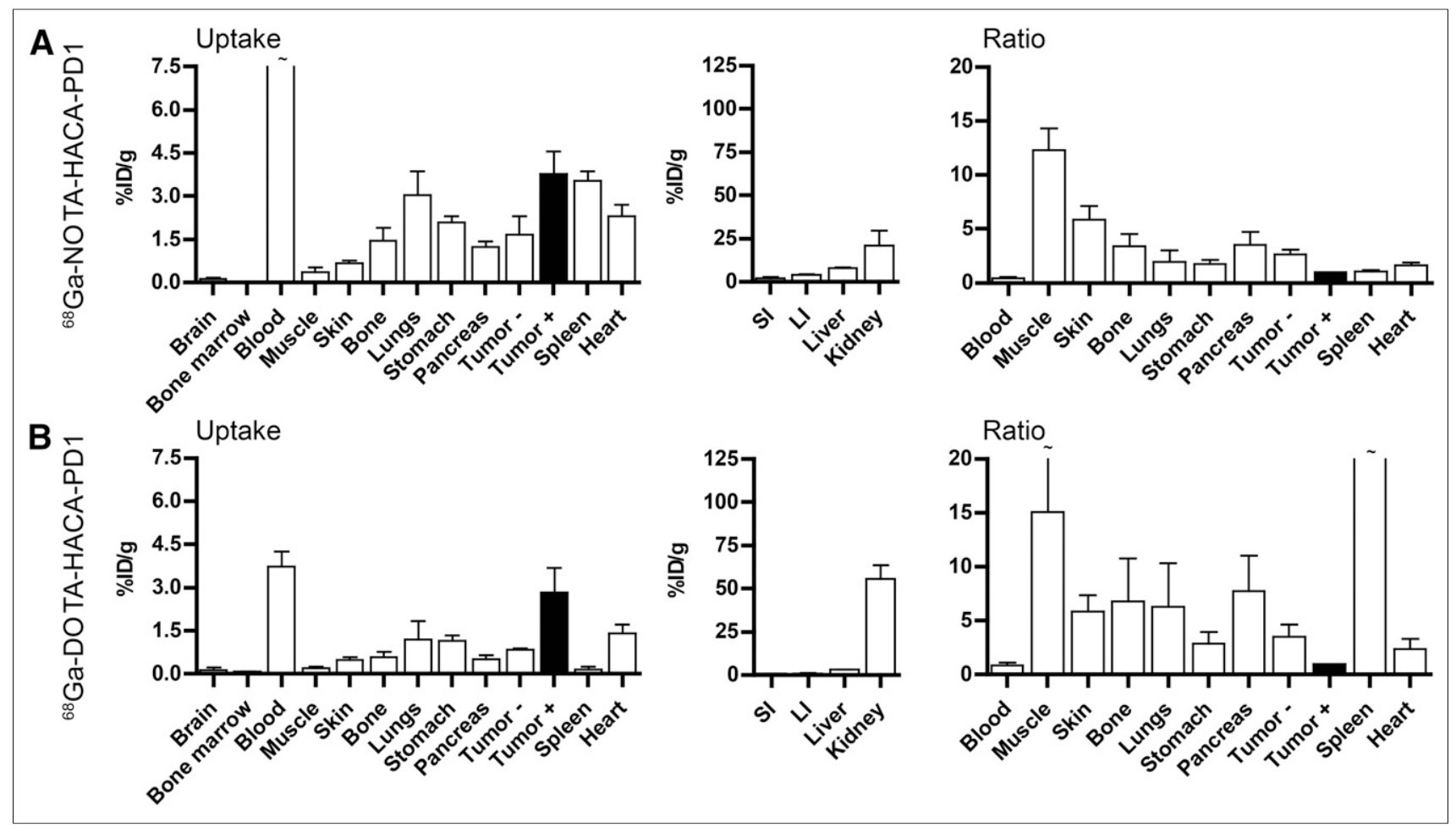

FIGURE 5. ${ }^{68} \mathrm{Ga}$ immuno-PET radiotracer biodistribution. Uptake $(\% \mathrm{ID} / \mathrm{g})$ in tissues and clearance organs and target-to-background ratios for ${ }^{68} \mathrm{Ga}-\mathrm{NOTA}-\mathrm{HACA}-\mathrm{PD} 1$ (A) and ${ }^{68} \mathrm{Ga}-\mathrm{DOTA}-\mathrm{HACA}-\mathrm{PD} 1$ (B). Data are reported as mean \pm SEM for each cohort $(\sim$ data off-scale, refer to Supplemental Tables 2 and 3). $\mathrm{LI}=$ large intestine; $\mathrm{SI}=$ small intestine; $\mathrm{T}+=$ tumor positive; $\mathrm{T}-=$ tumor negative.

imaging properties and biodistribution, prompting us to test aglycosylated ${ }^{68} \mathrm{Ga}$ variants in our comparative dual-tumor model study. Both ${ }^{68} \mathrm{Ga}-\mathrm{NOTA}-\mathrm{HACA}-\mathrm{PD} 1$ and ${ }^{68} \mathrm{Ga}$-DOTA-HACAPD1 showed highly specific tumor uptake and high tumor-tomuscle ratios at $1 \mathrm{~h}$ after injection. Future studies seeking to translate these variants will have to address higher signals seen in the blood and will include serum stability experiments to rule out that the tracer is not rapidly degrading. Although NOTA is accepted as forming a more stable complex with ${ }^{68} \mathrm{Ga}$, in this instance DOTA actually appeared to provide improved in vivo properties with the HAC-PD1 vector, potentially due to differences in charge and other physical properties. These findings highlight the difficulty of predicting the best tracer design configuration a priori due to the large number of variables that must be considered when designing immuno-PET radiotracers.

Further complicating the design and translation of immuno-PET tracers, radiometal production and half-life must be considered. Although ${ }^{64} \mathrm{Cu}$ and ${ }^{89} \mathrm{Zr}$ are amenable to antibody labeling for immuno-PET because of their long half-lives (12.7 and $78.5 \mathrm{~h}$, respectively), the need for a medical cyclotron limits the number of facilities with access to these radiometals. ${ }^{68} \mathrm{Ga}$ has only a 1.1-h half-life but can be easily produced at most facilities using a generator. Thus for small, fast-clearing binders such as HAC$\mathrm{PD} 1,{ }^{68} \mathrm{Ga}$ may be the more suitable choice. Because of the ease of production, cost, and suitable pharmacologic properties for early immune checkpoint imaging of ${ }^{68} \mathrm{Ga},{ }^{68} \mathrm{Ga}-\mathrm{NOTA}-\mathrm{HACA}$ PD1 and ${ }^{68} \mathrm{Ga}-\mathrm{DOTA}-\mathrm{HACA}-\mathrm{PD} 1$ human PD-L1 immuno-PET radiotracers merit further investigation for clinical translation.

Here we evaluated a large number of immuno-PET tracers and design parameters at the $1-\mathrm{h}$ time point, to optimize early immune checkpoint imaging, which is more amenable to the clinical routine. Although seeking to emphasize the importance of comparative radiotracer studies and design optimization, this work also highlights their difficulties. It was important to assess the effects of chelate, glycosylation, and radiometal in parallel because of the potential for high intrastudy variability commonly arising from differences in animals, specific activity of the source radiometal, purity of the radiopharmaceutical, and experimental workflow. Performing multiple tracer studies on a given day and time point is technically demanding and expensive, and we were not able to perform all of the characterization we would have liked to in this work. In the future, we seek to optimize the specific activity of the ${ }^{68} \mathrm{Ga}$ variants and we plan to perform dynamic imaging studies with ${ }^{68} \mathrm{Ga}$-DOTA-HACA-PD1 and ${ }^{68} \mathrm{Ga}$-NOTA-HACA-PD1 to fully assess their pharmacokinetics as well as dose optimization. Appropriate mathematic models for analyzing checkpoint expression in vivo are also needed and under development. \% ID/g is only a semiquantitative measure and does not capture the nonlinear binding mechanics of receptor ligand interactions. Partial-volume effects can also substantially affect activity estimates of small lesions. In this study, comparison of tracer ROI analysis with or without PVC showed relatively good agreement with the ${ }^{64} \mathrm{Cu}$ immuno-PET radiotracer uptake values measured by biodistribution. It is crucial that standardized calibration and quantitation methods are adopted, so that accurate models can be built to assess immune checkpoint expression.

Taken together, this work furthers our understanding of radiotracer design parameters, supports the importance of comparative radiotracer studies, and provides strategies for the development of optimized immuno-PET variants for human immune checkpoint 


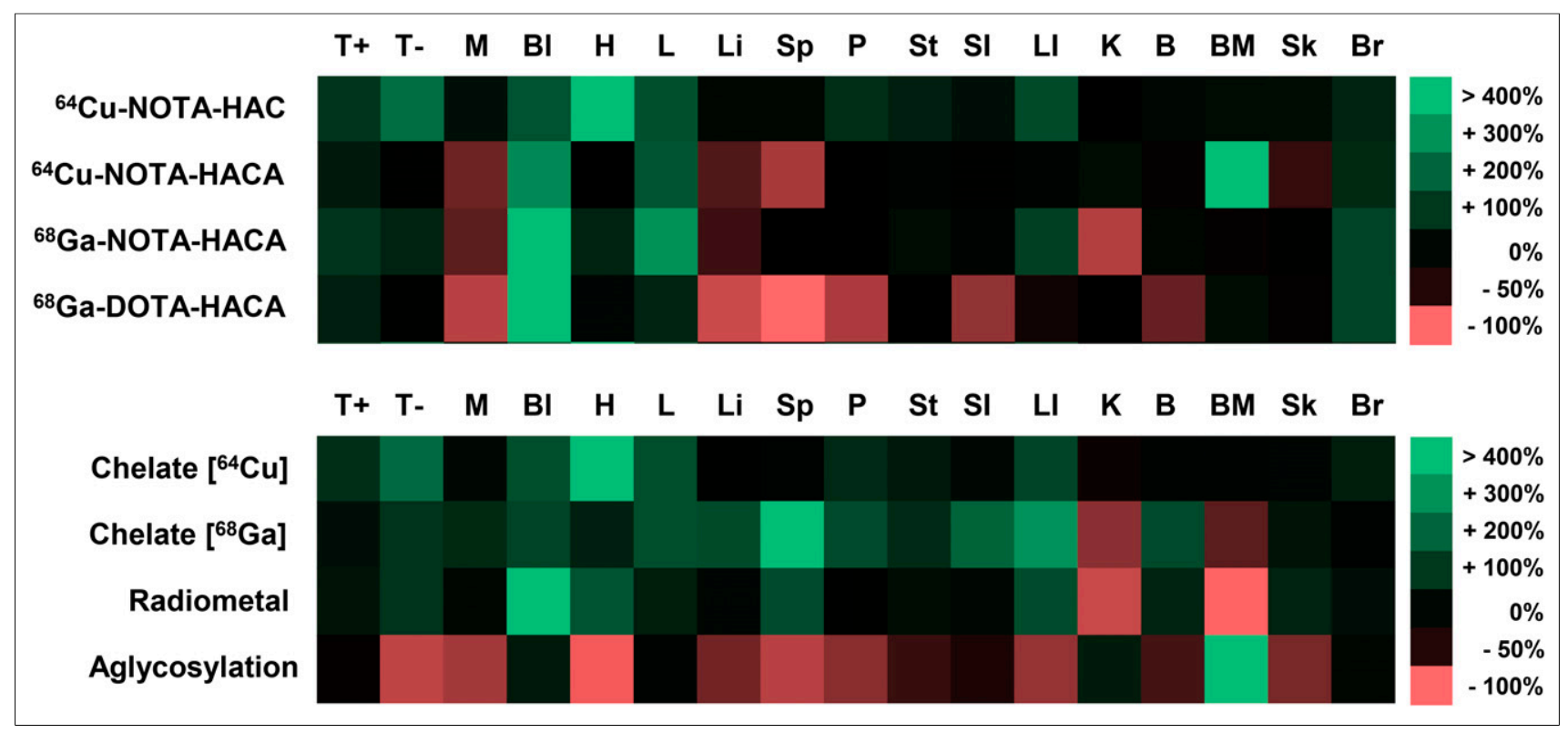

FIGURE 6. immuno-PET design decisions and outcomes. Heat map reflects relative changes in organ radiotracer uptake given design change. Scale bar indicates bright green as percentage increase in signal $>400 \%$ whereas bright red indicates $100 \%$ loss in signal. Optimal design modification would appear as green increase in signal for target (T+ = hPD-L1-positive tumor) and red decrease in signal for all off-target organs (T-, hPD-L1-negative tumor). $\mathrm{Bl}=$ blood; $\mathrm{B}=$ bone; $\mathrm{BM}=$ bone marrow; $\mathrm{Br}=$ brain; $\mathrm{H}=$ heart; $\mathrm{K}=$ kidney; $\mathrm{L}=$ lung; $\mathrm{Li}=$ liver; $\mathrm{LI}=$ large intestine; $\mathrm{M}=$ muscle; $\mathrm{P}=$ pancreas; $\mathrm{SI}=$ small intestine; $\mathrm{Sk}=$ skin; $\mathrm{Sp}=$ spleen; $\mathrm{St}=$ stomach. Top panel shows all radiotracer variants compared with ${ }^{64} \mathrm{Cu}-\mathrm{DOT} A-\mathrm{HAC}-\mathrm{PD} 1$, and bottom panel shows evaluation of effects of common immuno-PET design modifications:

$$
\begin{aligned}
& \text { chelate }{ }^{64} \mathrm{Cu}\left[\% \text { change }=100 \times \frac{\left({ }^{64} \mathrm{Cu}-\text { NOTA }- \text { HAC }- \text { PD1 }\right)-\left({ }^{64} \mathrm{Cu}-\text { DOTA }- \text { HAC }- \text { PD } 1\right)}{\left({ }^{64} \mathrm{Cu}-\text { DOTA }-\mathrm{HAC}-\mathrm{PD} 1\right)}\right] \\
& \text { chelate }{ }^{68} \mathrm{Ga}\left[\% \text { change }=100 \times \frac{\left({ }^{68} \mathrm{Ga}-\text { NOTA }- \text { HACA }- \text { PD } 1\right)-\left({ }^{68} \mathrm{Ga}-\text { DOTA }- \text { HACA }- \text { PD } 1\right)}{\left({ }^{68} \mathrm{Ga}-\text { DOTA }- \text { HACA }- \text { PD } 1\right)}\right] \\
& \text { glycosylation }\left[\% \text { change }=100 \times \frac{\left({ }^{64} \mathrm{Cu}-\text { NOTA }- \text { HACA }- \text { PD } 1\right)-\left({ }^{64} \mathrm{Cu}-\text { NOTA }- \text { HAC }- \text { PD } 1\right)}{\left({ }^{64} \mathrm{Cu}-\mathrm{NOTA}-\mathrm{HAC}-\mathrm{PD} 1\right)}\right] \\
& \text { radiometal }\left[\% \text { change }=100 \times \frac{\left({ }^{68} \mathrm{Ga}-\mathrm{NOTA}-\mathrm{HACA}-\mathrm{PD} 1\right)-\left({ }^{64} \mathrm{Cu}-\mathrm{NOTA}-\mathrm{HACA}-\mathrm{PD} 1\right)}{\left({ }^{64} \mathrm{Cu}-\mathrm{NOTA}-\mathrm{HACA}-\mathrm{PD} 1\right)}\right] .
\end{aligned}
$$

imaging. immuno-PET imaging could provide the tools necessary to validate disease biomarkers for checkpoint blockade, stratify patients for clinical trials, and ultimately improve cancer immunotherapies.

\section{CONCLUSION}

Here we report on 6 immuno-PET tracers for imaging human PD-L1 as well as discuss important design considerations and their relative effects on tracer biodistribution. These data provide practical guidance for future immuno-PET radiotracer design and optimization. We anticipate that small high-affinity engineered proteins such as HAC-PD1 will eventually be used to predict and monitor responders to checkpoint-based cancer immunotherapies in the clinic.

\section{DISCLOSURE}

We acknowledge the support of Kenneth Lau, Frezghi Habte, the Canary Foundation, and the Ben and Catherine Ivy Foundation. This material is based on work supported by the National Science Foundation Graduate Research Fellowship under grant no. DGE-114747 and the NIH TBi2 training grant (no. 2T32EB009653-06). Aaron T. Mayer, Roy L. Maute, Sydney R. Gordon, Melissa N. McCracken, and Irving L. Weissman are inventors of patents related to the high-affinity PD-1 proteins described in this manuscript. Sydney R. Gordon provides paid consulting services for Ab Initio Biotherapeutics, Inc., which licensed the aforementioned patents. Aaron M. Ring and Roy L. Maute are founders of Ab Initio Biotherapeutics, Inc. No other potential conflict of interest relevant to this article was reported.

\section{ACKNOWLEDGMENTS}

Small-animal PET/CT imaging and $\gamma$-counter measurements were performed in the SCi3 Stanford Small Animal Imaging Service Center.

\section{REFERENCES}

1. Nguyen LT, Ohashi PS. Clinical blockade of PD1 and LAG3, potential mechanisms of action. Nat Rev Immunol. 2015;15:45-56.

2. Bordon Y. Checkpoint parley. Nat Rev Cancer. 2015;15:3. 
3. Le Mercier I, Lines JL, Noelle RJ. Beyond CTLA-4 and PD-1, the generation $\mathrm{Z}$ of negative checkpoint regulators. Front Immunol. 2015;6:418.

4. Mellman I, Coukos G, Dranoff G. Cancer immunotherapy comes of age. Nature. 2011;480:480-489.

5. Scott AM, Wolchok JD, Old LJ. Antibody therapy of cancer. Nat Rev Cancer. 2012;12:278-287.

6. Korman AJ, Peggs KS, Allison JP. Checkpoint blockade in cancer immunotherapy. Adv Immunol. 2006;90:297-339.

7. Mahoney KM, Freeman GJ, McDermott DF. The next immune-checkpoint inhibitors: PD-1/PD-L1 blockade in melanoma. Clin Ther. 2015;37:764782.

8. Champiat S, Lambotte O, Barreau E, et al. Management of immune checkpoint blockade dysimmune toxicities: a collaborative position paper. Ann Oncol. 2016;27:559-574.

9. Wolchok JD, Kluger H, Callahan MK, et al. Nivolumab plus ipilimumab in advanced melanoma. N Engl J Med. 2013;369:122-133.

10. Brahmer JR, Tykodi SS, Chow LQM, et al. Safety and activity of anti-PD-L1 antibody in patients with advanced cancer. N Engl J Med. 2012;366:24552465.

11. Postel-Vinay S, Aspeslagh S, Lanoy E, Robert C, Soria JC, Marabelle A. Challenges of phase 1 clinical trials evaluating immune checkpoint targeted antibodies. Ann Oncol. 2016;27:214-224.

12. Zhang L, Qiu M, Jin Y, et al. Programmed cell death ligand 1 (PD-L1) expression on gastric cancer and its relationship with clinicopathologic factors. Int J Clin Exp Pathol. 2015;8:11084-11091.

13. Ngiow SF, Young A, Jacquelot N, et al. A threshold level of intratumor CD8 $+\mathrm{T}$ cell PD1 expression dictates therapeutic response to anti-PD1. Cancer Res. 2015;75:3800-3811.

14. Tamura T, Ohira M, Tanaka H, et al. Programmed death-1 ligand-1 (PDL1) expression is associated with the prognosis of patients with stage II/III gastric cancer. Anticancer Res. 2015;35:5369-5376.

15. Patel SP, Kurzrock R. PD-L1 expression as a predictive biomarker in cancer immunotherapy. Mol Cancer Ther. 2015;14:847-856.

16. Mahoney KM, Atkins MB. Prognostic and predictive markers for the new immunotherapies. Oncology. 2014;28:39-48.

17. Herbst RS, Soria JC, Kowanetz M, et al. Predictive correlates of response to the anti-PD-L1 antibody MPDL3280A in cancer patients. Nature. 2014;515:563567.

18. Meng X, Huang Z, Teng F, et al. Predictive biomarkers in PD-1/PD-L1 checkpoint blockade immunotherapy. Cancer Treat Rev. 2015;41:868-876.
19. Darb-Esfahani S, Kunze CA, Kulbe H, et al. Prognostic impact of programmed cell death-1 (PD-1) and PD-ligand 1 (PD-L1) expression in cancer cells and tumor-infiltrating lymphocytes in ovarian high grade serous carcinoma. Oncotarget. 2016;7:1486-1499.

20. Wu P, Wu D, Li L, Chai Y, Huang J. PD-L1 and survival in solid tumors: a metaanalysis. PLoS One. 2015;10:e131403.

21. Zhong A, Xing Y, Pan X, et al. Prognostic value of programmed cell death-ligand 1 expression in patients with non-small-cell lung cancer: evidence from an updated meta-analysis. Onco Targets Ther. 2015;8:3595-3601.

22. Wu AM, Senter PD. Arming antibodies: prospects and challenges for immunoconjugates. Nat Biotechnol. 2005;23:1137-1146.

23. Heskamp S, Hobo W, Molkenboer-Kuenen JD, et al. Non-invasive imaging of tumor PD-L1 expression using radiolabeled anti-PD-L1 antibodies. Cancer Res. 2015;75:2928-2936.

24. Josefsson A, Nedrow JR, Park S, et al. Imaging, biodistribution, and dosimetry of radionuclide-labeled PD-L1 antibody in an immunocompetent mouse model of breast cancer. Cancer Res. 2016;76:472-479.

25. Chatterjee S, Lesniak WG, Gabrielson M, Lisok A. A humanized antibody for imaging immune checkpoint ligand PD-L1 expression in tumors. Oncotarget. 2016;7:10215-10227.

26. Natarajan A, Mayer AT, Xu L, Reeves R, Gano J, Gambhir SS. A novel radiotracer for immunoPET imaging of PD-1 checkpoint expression on tumor infiltrating lymphocytes. Bioconjug Chem. 2015;26:2062-2069.

27. Maute RL, Gordon SR, Mayer T, et al. Engineering high-affinity PD-1 variants for optimized immunotherapy and immuno-PET imaging. Proc Natl Acad Sci USA. 2015;112:E6506-E6514.

28. Zeglis BM, Lewis JS. A practical guide to the construction of radiometallated bioconjugates for positron emission tomography. Dalton Trans. 2011;40:6168-6195.

29. Thurber GM, Schmidt MM, Wittrup KD. Antibody tumor penetration: transport opposed by systemic and antigen-mediated clearance. Adv Drug Deliv Rev. 2008;60:1421-1434.

30. Wilks MQ, Knowles SM, Wu AM, Huang S-C. Improved modeling of in vivo kinetics of slowly diffusing radiotracers for tumor imaging. J Nucl Med. 2014;55:1539-1544.

31. Wittrup KD, Thurber GM, Schmidt MM, Rhoden JJ. Practical theoretic guidance for the design of tumor-targeting agents. Methods Enzymol. 2012;503:255-268.

32. Price EW, Orvig C. Matching chelators to radiometals for radiopharmaceuticals. Chem Soc Rev. 2014;43:260-290.

33. Medzihradszky KF, Spencer DIR, Sharma SK, et al. Glycoforms obtained by expression in Pichia Pastoris improve cancer targeting potential of a recombinant antibody-enzyme fusion protein. Glycobiology. 2004;14:27-37. 